\title{
The Effect of Cycloplegia on the Ocular Biometric and Anterior Segment Parameters: A Cross-Sectional Study
}

\author{
Hamed Momeni-Moghaddam • Nasim Maddah • James S. Wolffsohn • \\ Mohammad Etezad-Razavi · Siamak Zarei-Ghanavati · Amir Akhavan Rezayat • \\ Majid Moshirfar
}

Received: March 17, 2019 / Published online: May 3, 2019

(C) The Author(s) 2019

\section{ABSTRACT}

Introduction: To evaluate the effects of cycloplegia on the biometric components and anterior segment parameters of the eye.

Methods: In this cross-sectional study, changes to axial length (AL), anterior chamber depth (ACD) lens thickness, anterior chamber angle (ACA) and volume, corneal thickness in the pupil center (PC), corneal curvature (CC) and white-to-white (WTW) following cycloplegia induced by tropicamide $1 \%$ in 42 eyes of patients aged 23-58 years were assessed. Biometric components and anterior segment

Enhanced Digital Features To view enhanced digital features for this article, go to https://doi.org/10.6084/ m9.figshare.7999433.

H. Momeni-Moghaddam

Health Promotion Research Center, Zahedan

University of Medical Sciences, Zahedan, Iran

H. Momeni-Moghaddam · N. Maddah

Department of Optometry, School of Paramedical

Sciences, Mashhad University of Medical Sciences,

Mashhad, Iran

J. S. Wolffsohn

Ophthalmic Research Group, Aston University, Life and Health Sciences, Birmingham, UK

M. Etezad-Razavi · S. Zarei-Ghanavati ·

A. Akhavan Rezayat

Eye Research Center, Mashhad University of

Medical Sciences, Mashhad, Iran parameters were measured using an IOLMaster 700 (Carl Zeiss Meditec, Jena, Germany) and a Pentacam HR (Oculus Optikgeräte $\mathrm{GmbH}$, Wetzlar, Germany), respectively.

Results: Significant statistical changes in ACD (increased by $0.06 \pm 0.05 \mathrm{~mm} ; p<0.001$ ), anterior chamber volume (increased by $15.19 \pm 10.32 \mathrm{~mm}^{3} ; p<0.001$ ), ACA (decreased by $\left.2.18 \pm 10.20^{\circ} ; p=0.029\right)$ and lens thickness (decreased by $0.02 \pm 0.03 \mathrm{~mm} ; p<0.001$ ) were observed post-cycloplegia, while the changes in $\mathrm{CC}$, corneal thickness in the PC, WTW and AL were not statistically different $(p>0.05)$. Also, a significant inferior displacement of the PC along the vertical axes was seen $(p=0.020)$.

Conclusion: Cycloplegia resulted in a deeper ACD and thinner lens thickness. These changes should be considered in determining intraocular lens (IOL) power to prevent refractive

\section{Moshirfar ( $\square)$}

Department of Ophthalmology and Visual Sciences, John A. Moran Eye Center, School of Medicine, University of Utah, Salt Lake City, USA e-mail: cornea2020@me.com

M. Moshirfar

Utah Lions Eye Bank, Murray, UT, USA

\section{Moshirfar}

HDR Research Center, Hoopes Vision, 11820 S. State Street Suite \#200, Draper, UT 84020, USA 
surprises in cataract surgery and also in the phakic IOL implantation.

$\begin{array}{lllr}\text { Keywords: } & \text { Anterior } & \text { segment; } & \text { Biometry; } \\ \text { Crystalline } & \text { lens; } & \text { Cycloplegia; } & \text { Ocular } \\ \text { components } & & & \end{array}$

\section{INTRODUCTION}

Nowadays, cycloplegic agents such as tropicamide, homatropine and cyclopentolate have diagnostic and therapeutic applications. These clinical applications include fundus examination, anterior segment assessment, cycloplegic refraction, treatment of uveitis, amblyopia treatment via pharmacological penalization [1], and myopia control in children [2]. Detailed evaluation of the anterior segment is important for corneal refractive surgery, diagnosis and follow-up in patients with glaucoma, intraocular lens (IOL) calculation, and in determining effective lens position in cataract surgery and phakic IOL implantation (pIOL) [1, 3, 4]. Accurate measurement of axial length (AL), anterior chamber depth (ACD) and corneal curvature (CC) is essential for the calculation of IOL power according to different formulas $[5,6]$. In addition, white-to-white (WTW) and lens thickness (LT) are necessary items for formulas such as the Holladay II IOL power prediction formula [6]. For example, overestimation of ACD may result in corneal endothelial cell damage [3, 4]. Also, accurate cycloplegic refraction, CC assessment and measurement of central corneal thickness (CCT) are essential in pre- and postoperative assessment of corneal refractive surgery [7]. Assessing ACD is important to determine whether there is enough room for the pIOLs, and the angle dimensions are important especially for an anterior chamber angle (ACA) fixated lens [8]. It is evident that a shallow ACD and/or narrow angle may increase the risk of iris pigment chaffing, particularly when posterior chamber hyperopic pIOLs are implanted [9].

Although several studies have investigated the effects of cycloplegia on the anterior segment parameters and biometry components of the eyes, there are some controversies about the changes in these parameters following cycloplegia $[1,4,5,10-12]$; a summary of the literature review is presented in Table 1.

This study was designed to assess these variations following induced temporary paralysis of the ciliary muscle and iris sphincter by the cycloplegic agent, tropicamide $1 \%$.

\section{METHODS}

In this cross-sectional study, 42 eyes of 42 patients with an age range of $23-58$ years were assessed. All steps of this study followed the tenets of the Declaration of Helsinki. In addition, the study was approved by the ethics committee of Mashhad University of Medical Sciences. Inclusion criteria were clear central corneas and crystalline lens with no ocular and systemic diseases. Exclusion criteria included history of corneal excimer laser refractive surgery, keratoconus, pellucid marginal degeneration, high myopia $6 \mathrm{D}$ or higher and history of ocular trauma.

The refractive status [sphere, cylinder, axis and spherical equivalent (SE)], anterior segment parameters and the ocular biometry were determined using a Topcon auto-kerato-refractometer (Topcon Corp., Tokyo, Japan), Pentacam HR (Oculus Optikgeräte $\mathrm{GmbH}$, Wetzlar, Germany) and IOLMaster 700 (Carl Zeiss Meditec, Jena, Germany), respectively. The evaluations were repeated after producing the cycloplegic effect. For this purpose, one drop of tropicamide $1 \%$ was instilled every $5 \mathrm{~min}$ for three applications, and the measurements were repeated $30 \mathrm{~min}$ after the last drop.

The assessed anterior segment parameters determined using the Pentacam included external ACD (measured from the epithelium), ACA and anterior chamber volume (ACV), corneal thickness at the pupil center (PC) associated with geometric positions of the PC along the horizontal $(x)$ and vertical $(y)$ axes with respect to the corneal apex as the reference point and the corneal diameter (WTW). Ocular biometric parameters included AL, ACD measured from the epithelium and LT. Mean keratometry reading $(\mathrm{KRm})$, and the magnitude and axis of corneal astigmatism (CA) were determined using all three devices, the auto- 


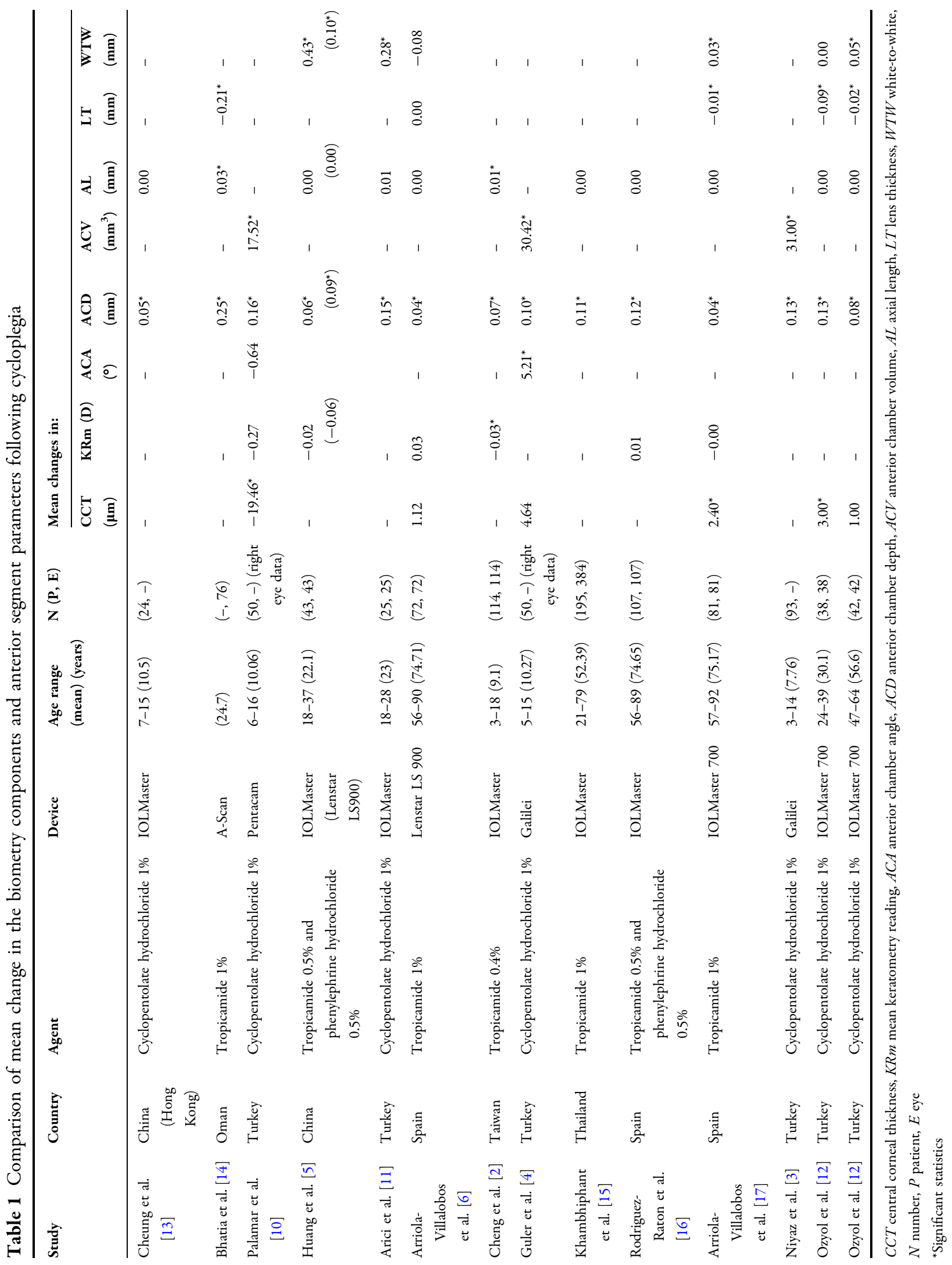


kerato-refractometer, the Pentacam and the IOLMaster 700. The vector analysis method was used to determine the astigmatic difference between the two states, in which the spherocylindrical refraction and CA were transformed to a Jackson crossed-cylinder format, and the refractive data ( $C$ : cylinder; $\alpha$ : cylinder axis) were used to calculate $J 0$ and $J 45$, where $J 0=(-C / 2) \cos (2 \alpha)$, and $J 45=(-C / 2) \sin (2 \alpha)$.

To compute the astigmatic difference in the two states, the difference between $J 0$ and $J 45$ of the first- and second-state astigmatism was calculated separately. To better understand astigmatic changes, the values of power vector were transformed to the conventional format by applying the following formula:

$$
C=-2 \sqrt{ }(J 0)^{2}+(J 45)^{2} .
$$

All measurements were done under the supervision of an experienced optometrist who did not have access to the pre-cycloplegic data, in order to prevent any bias or error. All measurements were performed between 6 and 8 p.m.

Assessment of the normality of data using the Kolmogorov-Smirnov test showed no normal distribution. Data were analyzed in SPSS 22 software (IBM Corp., Armonk, NY, USA) using the Wilcoxon signed-rank, Kruskal-Wallis and Mann-Whitney $U$ tests. The significance level was considered to be 0.05 .

\section{RESULTS}

In this study, 42 eyes of 42 patients (28 female and 14 male) were evaluated. The mean age was $35.2 \pm 9.4$, with a range of $23-58$ years.

Table 2 presents the refractive status before and after cycloplegia.

The mean difference seen in the sphere, cylinder, axis and SE was $0.32 \pm 0.26 \mathrm{D}$, $0.11 \pm 0.44 \mathrm{D}, 8.70^{\circ} \pm 53.82^{\circ}$ and $0.22 \pm 0.20$ $\mathrm{D}$, respectively. Only a significant hyperopic shift was observed in the spherical component $(p<0.001)$ and SE $(p<0.001)$, while the magnitude and axis of the refractive cylinder did not change significantly following cycloplegia.

Regarding the anterior segment parameters obtained using the Pentacam, cycloplegia induced statistically significant changes in external ACD $(p<0.001)$, ACV $(p<0.001)$, ACA $(p=0.029)$ and the PC along the $y$-axis $(p=0.020)$. The ACD and ACV increased, while the ACA decreased post-cycloplegia, with a mean difference of $0.10 \pm 0.05 \mathrm{~mm}$, $15.19 \pm 10.32 \mathrm{~mm}^{3}$ and $-2.18 \pm 10.20^{\circ}$, respectively. The PC showed no significant change the $x$-axis, while a statistically significant change of $0.06 \pm 0.17 \mathrm{~mm}$ along the $y$-axis and in the negative direction was observed in comparisons of the locations before and after cycloplegia (Table 3 ).

Table 4 shows the mean and standard deviation of the ocular biometry components obtained from the IOLMaster 700 before and after cycloplegia. The mean changes in $\mathrm{AL}, \mathrm{ACD}$ and LT were $0.0 \pm 0.01,0.06 \pm 0.05$ and $-0.02 \pm 0.03 \mathrm{~mm}$, respectively, with no significant change in AL, while ACD significantly increased and LT decreased following cycloplegia.

Comparison of the changes in ocular biometry and anterior segment parameters separately in the two age groups, $<40$ and $\geq 40$ years, only showed a significant difference in LT changes $(p=0.016)$ (Table 5).

Table 6 presents the mean and standard deviation of the mean $\mathrm{KRm}$ and CA using these three devices. A comparison of the mean KRm and CA (magnitude and axis) obtained using these three devices separately pre- and post-cycloplegia showed no significant difference using the Kruskal-Wallis test $(p>0.05)$. Also, the paired comparison of these variables was not significantly different separately for each device before and after use of tropicamide using the Wilcoxon signed-rank test $(p>0.05)$.

A comparison of ACD measured using the Pentacam HR and IOLMaster 700 showed no significant difference in mean ACD between the two devices using the Mann-Whitney $U$ test separately for before $(p=0.697)$ and after $(p=0.381)$ cycloplegia.

\section{DISCUSSION}

The results of this study showed significant increase in ACD and ACV, while ACA and LT 
Table 2 Mean and standard deviation (SD) of the sphere, cylinder power/axis and spherical equivalent (SE) before and after cycloplegia $(n=42$ eyes)

\begin{tabular}{lllc}
\hline Variables & \multicolumn{2}{l}{ Mean \pm SD $(\mathbf{9 5 \%}$ CI $)$} & Wilcoxon signed-rank test \\
\cline { 2 - 3 } & Before cycloplegia & After cycloplegia & \\
\hline Sphere (D) & $-3.23 \pm 3.68(-4.37,-2.08)$ & $-2.90 \pm 3.68(-4.05,-1.75)$ & $<0.001$ \\
Cylinder (D) & $-1.44 \pm 1.15(-1.80,-1.08)$ & $-1.32 \pm 1.12(-1.67,-0.96)$ & 0.139 \\
Axis $\left(^{\circ}\right)$ & $95.22 \pm 64.24(74.94,115.50)$ & $86.51 \pm 65.81(65.74,107.29)$ & 0.604 \\
SE (D) & $-2.33 \pm 1.82(-2.90,-1.76)$ & $-2.11 \pm 1.82(-2.68,-1.54)$ & $<0.001$ \\
\hline
\end{tabular}

Table 3 Mean and standard deviation (SD) of anterior segment parameters using the Pentacam before and after cycloplegia $(n=42$ eyes $)$

\begin{tabular}{llll}
\hline Variables & Mean \pm SD $(95 \%$ CI $)$ & Wilcoxon signed-rank \\
\cline { 2 - 3 } & Pre-cycloplegia & Post-cycloplegia & test \\
\hline ACD $(\mathrm{mm})$ & $3.63 \pm 0.34(3.52,3.74)$ & $3.73 \pm 0.35(3.62,3.84)$ & $<0.001$ \\
ACA $\left(^{\circ}\right)$ & $40.08 \pm 6.70(37.99,42.17)$ & $37.89 \pm 10.69(34.56,41.22)$ & 0.029 \\
ACV $\left(\mathrm{mm}^{3}\right)$ & $180.69 \pm 34.64(169.89$, & $195.88 \pm 33.20(185.53$, & $<0.001$ \\
& $191.48)$ & $206.22)$ & \\
Pachy. @ pupil center & $526.33 \pm 38.82(514.23$, & $527.42 \pm 41.15(514.60$, & 0.066 \\
$(\mu \mathrm{m})$ & $538.43)$ & $540.25)$ & \\
PC $x(\mathrm{~mm})$ & $-0.01 \pm 0.18(-0.07,0.03)$ & $0.00 \pm 0.20(-0.05,0.06)$ & 0.101 \\
PCy $(\mathrm{mm})$ & $0.01 \pm 0.13(-0.02,0.06)$ & $-0.04 \pm 0.15(-0.09,0.00)$ & 0.020 \\
WTW $(\mathrm{mm})$ & $11.94 \pm 0.42(11.80,12.07)$ & $11.98 \pm 0.52(11.82,12.15)$ & 0.123 \\
\hline
\end{tabular}

$A C D$ anterior chamber depth, $A C A$ anterior chamber angle, $A C V$ anterior chamber volume, $P C x / P C y$ geometric positions of the pupil center along the coordinate axes, $W T W$ white-to-white

decreased significantly after producing cycloplegia using tropicamide $1 \%$. Another point was significant displacement of the PC along the vertical axes in the negative direction or inferiorly.

As reported, ACD is an important factor in IOL power calculation and diagnosis of primary angle closure glaucoma (PACG), so imprecise measurement may lead to undesirable refractive errors after cataract surgery, reprieve PACG diagnosis and corneal endothelial cell damage following pIOL $[1,3,14]$. The results of this study show a significant increase in ACD and ACV and a decrease in LT following cycloplegia. The results confirm previous studies that reported a significant increase in ACD and ACV after instillation of cycloplegic and mydriatic drops [1, 3, 4, 10]. Rodriguez-Raton et al. [16] found that $\mathrm{ACD}$ and IOL, calculated based on the Haigis formula (ACD is used in this formula), were significantly changed after application of tropicamide and phenylephrine, whereas the IOL calculation based on the SRK/T formula was not affected.

Similar to the present findings, several studies reported a reduction in LT after cycloplegia $[14,17,18]$. Ocular accommodation increases LT and moves the lens forward [19], while temporary paralysis of the ciliary muscle leads to an increase in ACD by reducing the crystalline lens curvature (flattening) and backward displacement of its geometrical center [7, 12]. 
Table 4 Mean and standard deviation (SD) of ocular biometry components using the IOLMaster 700 before and after cycloplegia $(n=42$ eyes)

\begin{tabular}{llll}
\hline Variables & \multicolumn{2}{l}{ Mean \pm SD $(\mathbf{9 5 \%} \mathbf{C I})$} & Wilcoxon signed-rank test \\
\cline { 2 - 3 } & Pre-cycloplegia & Post-cycloplegia & \\
\hline AL $(\mathrm{mm})$ & $24.91 \pm 1.45(24.46,25.36)$ & $24.91 \pm 1.45(24.45,25.36)$ & 0.376 \\
ACD $(\mathrm{mm})$ & $3.60 \pm 0.32(3.49,3.70)$ & $3.66 \pm 0.34(3.55,3.77)$ & $<0.001$ \\
LT $(\mathrm{mm})$ & $3.76 \pm 0.28(3.67,3.85)$ & $3.74 \pm 0.28(3.65,3.83)$ & $<0.001$ \\
\hline
\end{tabular}

$A L$ axial length, $A C D$ anterior chamber depth, $L T$ lens thickness

Table 5 Mean and standard deviation (SD) of changes in the ocular biometry and anterior segment parameters separately in two age groups $(n=42$ eyes)

\begin{tabular}{llll}
\hline Age group component & $\begin{array}{l}<\mathbf{4 0} \text { years }(\boldsymbol{n}=\mathbf{3 0} \text { eyes }) \\
\text { Mean } \pm \mathbf{S D}(\mathbf{9 5 \%} \mathbf{C I})\end{array}$ & $\begin{array}{l}\mathbf{2} \text { 40 years }(\boldsymbol{n}=\mathbf{1 2} \text { eyes }) \\
\text { Mean } \pm \mathbf{S D}(\mathbf{9 5 \%} \mathbf{C I})\end{array}$ & Mann-Whitney $\boldsymbol{U}$ test \\
\hline AL change $(\mathrm{mm})$ & $0.00 \pm 0.01(0.00,0.00)$ & $0.00 \pm 0.00(0.00,0.00)$ & 0.068 \\
ACD change $(\mathrm{mm})$ & $0.07 \pm 0.03(0.06,0.08)$ & $0.03 \pm 0.08(-0.01,0.09)$ & 0.945 \\
LT change $(\mathrm{mm})$ & $-0.03 \pm 0.02(-0.04,-0.02)$ & $0.00 \pm 0.03(-0.02,0.02)$ & 0.016 \\
ACA change $\left({ }^{\circ}\right)$ & $-2.41 \pm 11.43(-6.68,1.85)$ & $-1.61 \pm 6.53(-5.77,2.53)$ & 0.417 \\
ACV change $\left(\mathrm{mm}^{3}\right)$ & $16.03 \pm 10.23(12.21,19.85)$ & $13.08 \pm 10.70(6.28,19.88)$ & 0.731 \\
\hline
\end{tabular}

$A L$ axial length, $A C D$ anterior chamber depth, $L T$ lens thickness, $A C A$ anterior chamber angle, $A C V$ anterior chamber volume

Higashiyama et al. [18] reported a significant correlation between the changes in LT and ACD. In contrast to the current study, ArriolaVillalobos et al. [6] reported that changes in LT were not significant after instillation of tropicamide $1 \%$ despite a significant increase in ACD. This discrepancy may be attributed to the difference in subject age between these two studies $(74.7 \pm 7.5$ years vs. $35.2 \pm 9.4$ years in the present study). Although a decrease in the elasticity of the crystalline lens capsule in elderly populations may affect the response to the cycloplegic agents [12], a similar reduction in LT following cycloplegia by tropicamide $1 \%$ has been found in an elderly patients $(75.2 \pm 7.5$ years) [17].

The unremarkable effect of cycloplegia on $\mathrm{AL}$ in the present study confirms previous studies that showed no significant change in this parameter following mydriasis and/or cycloplegia $[7,11,13,15,20]$. On the contrary, there are studies that reported statistically, but not clinically, significant changes in AL following cycloplegia induced by homatropine $2 \%$ and cyclopentolate $1 \%$ [14]. Similar findings of a significant increase in $\mathrm{AL}$ were reported after application of $0.4 \%$ tropicamide [2] and in hyperopic eyes, although a considerable reduction was obtained in myopic eyes [21].

Contrary to our research results, Chang et al. [7], Arici et al. [11] and Arriola-Villalobos et al. [17] noted significant widening of WTW after the application of a cycloplegic, although these changes appear to be unverifiable.

In the present study, the change in corneal thickness in the PC was not significant, which is in line with previous reports $[1,6,18]$. In contrast to the present findings, however, a significant increase in CCT was cited in the measurement of the effects of cycloplegia on anterior segment parameters with anterior segment optical coherence tomography (AS-OCT) and a Pentacam; this change was attributed to the possible role of reflex tearing and not 
Table 6 Mean and standard deviation (SD) of mean keratometry (KRm), magnitude and axis of corneal astigmatism (CA) using the Topcon auto-kerato-refractometer, the Pentacam HR and the IOLMaster 700 ( $n=42$ eyes)

\begin{tabular}{|c|c|c|c|c|}
\hline \multirow[t]{2}{*}{ Variables } & \multicolumn{3}{|l|}{ Mean \pm SD $(95 \% \mathrm{CI})$} & \multirow{2}{*}{$\begin{array}{l}\text { Kruskal-Wallis } \\
\text { test }\end{array}$} \\
\hline & $\begin{array}{l}\text { Topcon auto-kerato- } \\
\text { refractometer }\end{array}$ & Pentacam HR & IOLMaster 700 & \\
\hline \multicolumn{5}{|l|}{ Mean KRm (D) } \\
\hline Pre-cycloplegia & $43.49 \pm 2.38(42.68,44.30)$ & $\begin{array}{l}43.13 \pm 3.17(42.14 \\
\quad 44.12)\end{array}$ & $\begin{array}{l}43.63 \pm 2.82(42.75 \\
\quad 44.51)\end{array}$ & 0.558 \\
\hline Post-cycloplegia & $43.46 \pm 2.37(42.65,44.26)$ & $\begin{array}{l}43.15 \pm 3.09(42.19 \\
\quad 44.12)\end{array}$ & $\begin{array}{l}43.69 \pm 2.87(42.78 \\
44.59)\end{array}$ & 0.566 \\
\hline $\begin{array}{l}\text { Wilcoxon signed- } \\
\text { rank test }\end{array}$ & 0.139 & 0.362 & 0.145 & - \\
\hline \multicolumn{5}{|l|}{ CA (D) } \\
\hline Pre-cycloplegia & $1.46 \pm 1.04(1.11,1.81)$ & $\begin{array}{l}1.53 \pm 0.98(1.22 \\
1.84)\end{array}$ & $\begin{array}{l}1.55 \pm 1.03(1.23 \\
1.87)\end{array}$ & 0.803 \\
\hline Post-cycloplegia & $1.45 \pm 1.05(1.09,1.80)$ & $\begin{array}{c}1.52 \pm 0.99(1.21 \\
1.83)\end{array}$ & $\begin{array}{l}1.55 \pm 1.10(1.20 \\
1.90)\end{array}$ & 0.825 \\
\hline $\begin{array}{l}\text { Wilcoxon signed- } \\
\text { rank test }\end{array}$ & 0.825 & 0.745 & 0.148 & - \\
\hline \multicolumn{5}{|l|}{$\mathrm{CA}$ axis $\left(^{\circ}\right)$} \\
\hline Pre-cycloplegia & $\begin{array}{l}92.69 \pm 74.15(67.61 \\
117.78)\end{array}$ & $\begin{array}{l}94.14 \pm 29.25(85.02 \\
103.26)\end{array}$ & $\begin{array}{l}77.52 \pm 72.02(55.07 \\
99.96)\end{array}$ & 0.278 \\
\hline Post-cycloplegia & $\begin{array}{l}100.39 \pm 73.09(75.66 \\
125.12)\end{array}$ & $\begin{array}{l}92.40 \pm 31.33(82.63 \\
102.16)\end{array}$ & $\begin{array}{l}86.68 \pm 72.85(63.68 \\
09.68)\end{array}$ & 0.683 \\
\hline $\begin{array}{l}\text { Wilcoxon signed- } \\
\text { rank test }\end{array}$ & 0.745 & 0.107 & 0.251 & - \\
\hline
\end{tabular}

corneal swelling during measurements [7]. Also, some studies have reported a decrease in CCT in pre-presbyopic eyes [12] and in children after cycloplegia [10].

Some studies reported no significant change in mean $\mathrm{KRm}$ following cycloplegia, $[6,10,20]$, while others showed a statistically remarkable flattening effect $[2,7]$. This flattening was justified based on the paralysis of the ciliary muscle following cycloplegic agents which reduce its force on the sclera spur and lead to the corneal flattening [2]. Assessing the changes in the front and back CC following application of miotic (pilocarpine hydrochloride 2\%) and mydriatic (tropicamide/phenylephrine hydrochloride) agents showed significant steepening only in the back surface and significant flattening in both front and back surfaces, respectively [22]. Chang et al. [7] illustrated that flattening in the posterior CC was more in corneas with steeper posterior curvatures. The current study showed no significant change in the mean KRm using the Topcon auto-kerato-refractometer, Pentacam HR and IOLMaster 700 after cycloplegia.

With regard to ACA, the present study found a significant narrowing of the angle following cycloplegia, which is in contrast to the results of studies by Niyaz et al. and Guler et al., who reported significant widening of ACA [3, 4], confirming the findings of Arici et al. [1]. As the 
ACA value is one of the most important factors for diagnosis and follow-up of glaucoma, the effect of cycloplegia on ACA should be considered during examination of patients.

Assessing the changes in different ocular biometry and anterior segment parameters before 40 years and above of 40 years affirmed the previous study showing that the changes in LT decrease with age [23].

One limitation of this study was the evaluation of only myopic eyes; therefore, assessing these changes in hyperopic eyes and in a wide range of ages, including children, adults and the elderly, is proposed for future research. Another study limitation is the absence of a control group.

In conclusion, cycloplegia resulted in a deeper ACD and thinner LT. These changes should be considered in determining IOL power to prevent refractive surprises in cataract surgery and pIOL implantation.

\section{ACKNOWLEDGEMENTS}

The authors would like to thank the participants who made this study possible.

Funding. No funding or sponsorship was received for this study or publication of this article.

Authorship. All named authors meet the International Committee of Medical Journal Editors (ICMJE) criteria for authorship for this article, take responsibility for the integrity of the work as a whole, and have given their approval for this version to be published.

Disclosures. Hamed Momeni-Moghaddam, Nasim Maddah, James S. Wolffsohn, Mohammad Etezad-Razavi, Siamak Zarei-Ghanavati, Amir Akhavan Rezayat and Majid Moshirfar have nothing to declare.

Compliance with Ethics Guidelines. All procedures performed in studies involving human participants were in accordance with the ethical standards of the institutional research committee of Mashhad University of Medical Sciences and with the 1964 Helsinki Declaration and its later amendments or comparable ethical standards. Informed consent was obtained from all individual participants included in the study.

Data availability. The data in our manuscript will not be deposited.

Open Access. This article is distributed under the terms of the Creative Commons Attribution-NonCommercial 4.0 International License (http://creativecommons.org/licenses/ by-nc/4.0/), which permits any noncommercial use, distribution, and reproduction in any medium, provided you give appropriate credit to the original author(s) and the source, provide a link to the Creative Commons license, and indicate if changes were made.

\section{REFERENCES}

1. Arici C, Turk A, Ceylan OM, Kola M, Hurmeric V. Effects of $1 \%$ cyclopentolate hydrochloride on anterior segment parameters obtained with Pentacam in young adults. Arq Bras Oftalmol. 2014;77:228-32.

2. Cheng H-C, Hsieh Y-T. Short-term refractive change and ocular parameter changes after cycloplegia. Optom Vis Sci. 2014;91:1113-7.

3. Niyaz L, Can E, Seymen Z, Eraydin B. Comparison of anterior segment parameters obtained by dualScheimpflug analyzer before and after cycloplegia in children. J Pediatr Ophthalmol Strabismus. 2016;53:234-7.

4. Guler E, Guragac FB, Tenlik A, Yagci R, Arslanyilmaz Z, Balci M. Influences of topical cyclopentolate on anterior chamber parameters with a dualScheimpflug analyzer in healthy children. J Pediatr Ophthalmol Strabismus. 2015;52:26-30.

5. Huang J, McAlinden C, Su B, et al. The effect of cycloplegia on the lenstar and the IOLMaster biometry. Optom Vis Sci. 2012;89:1691-6.

6. Arriola-Villalobos P, Díaz-Valle D, Garzon N, et al. Effect of pharmacologic pupil dilation on OLCR optical biometry measurements for IOL predictions. Eur J Ophthalmol. 2014;24:53-7. 
7. Chang SW, Lo AY, Su PF. Anterior segment biometry changes with cycloplegia in myopic adults. Optom Vis Sci. 2016;93:12-8.

8. Gimbel HV, Norton NR, Amritanand A. Angle-supported phakic intraocular lenses for the correction of myopia: three-year follow-up. J Cataract Refract Surg. 2015;41:2179-89.

9. Kamiya K, Shimizu K, Igarashi A, et al. Posterior chamber phakic intraocular lens implantation: comparative, multicentre study in 351 eyes with low-to-moderate or high myopia. Br J Ophthalmol. 2018;102:177-81.

10. Palamar M, Egrilmez S, Uretmen O, Yagci A, Kose S. Influences of cyclopentolate hydrochloride on anterior segment parameters with Pentacam in children. Acta Ophthalmol. 2011;89:e461-5.

11. Arici C, Turk A, Ceylan OM, Kola M. The effect of topical 1\% cyclopentolate on IOLMaster biometry. Optom Vis Sci. 2014;91:1343-7.

12. Özyol P, Özyol E, Baldemir E. Changes in ocular parameters and intraocular lens powers in aging cycloplegic eyes. Am J Ophthalmol. 2017;173:76-83.

13. Cheung SW, Chan R, Cheng R, Cho P. Effect of cycloplegia on axial length and anterior chamber depth measurements in children. Clin Exp Optom. 2009;92:476-81.

14. Bhatia J. Effect of tropicamide and homatropine eye drops on A-scan parameters of the phakic normal eyes. Oman Med J. 2011;26:23-5.

15. Khambhiphant B, Chatbunchachai N, Pongpirul K. The effect of pupillary dilatation on IOL power measurement by using the IOLMaster. Int Ophthalmol. 2015;35:853-9.
16. Rodriguez-Raton A, Jimenez-Alvarez M, Arteche-Limousin L, Mediavilla-Pena E, Larrucea-Martinez I. Effect of pupil dilation on biometry measurements with partial coherence interferometry and its effect on IOL power formula calculation. Eur J Ophthalmol. 2015;25:309-14.

17. Arriola-Villalobos P, Almendral-Gomez J, Garzon N, et al. Effect of pharmacological pupil dilation on measurements and IOL power calculation made using the new swept-source optical coherence tomography-based optical biometer. J Fr Ophtalmol. 2016;39:859-65.

18. Higashiyama T, Iwasa $M$, Ohji M. Changes in the anterior segment after cycloplegia with a biometer using swept-source optical coherence tomography. PLoS One. 2017;12:e0183378.

19. Malyugin BE, Shpak AA, Pokrovskiy DF. Accommodative changes in anterior chamber depth in patients with high myopia. J Cataract Refract Surg. 2012;38:1403-7.

20. Adler G, Shahar J, Kesner R, et al. Effect of pupil size on biometry measurements using the IOLMaster. Am J Ophthalmol. 2015;159:940-4.

21. Gao L, Zhuo X, Kwok AK, Yu N, Ma L, Wang J. The change in ocular refractive components after cycloplegia in children. Jpn J Ophthalmol. 2002;46:293-8.

22. Saitoh K, Yoshida K, Hamatsu Y, Tazawa Y. Changes in the shape of the anterior and posterior corneal surfaces caused by mydriasis and miosis: detailed analysis. J Cataract Refract Surg. 2004;30:1024-30.

23. Shao Y, Tao A, Jiang H, et al. Age-related changes in the anterior segment biometry during accommodation. Investig Ophthalmol Vis Sci. 2015;56:3522-30. 